\title{
Full Aging in Spin Glasses
}

\author{
G. F. Rodriguez and G. G. Kenning \\ Department of Physics, University of California \\ Riverside, California 92521-0101 \\ R. Orbach \\ Department of Energy, Office of the Director of Science, \\ Washington $D C$
}

(Dated: November 13, 2018)

\begin{abstract}
The discovery of dynamic memory effects in the magnetization decays of spin glasses in 1983 marked a turning point in the study of the highly disordered spin glass state. Detailed studies of the memory effects have led to much progress in understanding the qualitative features of the phase space. Even so, the exact nature of the magnetization decay functions have remained elusive, causing confusion. In this letter, we report strong evidence that the Thermoremanent Magnetization (TRM) decays scale with the waiting time, $t_{w}$. By employing a series of cooling protocols, we demonstrate that the rate at which the sample is cooled to the measuring temperature plays a major role in the determination of scaling. As the effective cooling time, $t_{c}^{e f f}$, decreases, $\frac{t}{t_{w}}$ scaling improves and for $t_{c}^{e f f}<20 s$ we find almost perfect $\frac{t}{t_{w}}$ scaling, i.e full aging.

PACS numbers: $75.50 \mathrm{Lk}$
\end{abstract}

Since the discovery of aging effects in spin glasses approximately twenty years ago 1] 2], much effort has gone into determining the exact time dependence of the memory decay functions. In particular, memory effects show up in the Thermoremanent Magnetization (TRM) (or complementary Zero-Field Cooled (ZFC) magnetization) where the sample is cooled through its spin glass transition temperature in a small magnetic field (zero field) and held in that particular field and temperature configuration for a waiting time, $t_{w}$. At time $t_{w}$, a change in the magnetic field produces a very long time decay in the magnetization. The decay is dependent on the waiting time. Hence, the system has a memory of the time it spent in the magnetic field. A rather persuasive argument [3] suggests that for systems with infinite equilibration times, the decays must scale with the only relevant time scale in the experiment, $t_{w}$. This would imply that plotting the magnetization on a $\mathrm{t} / t_{w}$ axis would collapse the different waiting time curves onto each other. This effect has not been observed.

What has been observed [4] is that the experimentally determined magnetization decays will scale with a modified waiting time, $\left(t_{w}\right)^{\mu}$. Where $\mu$ is a fitting parameter. For $\mu<1$ the system is said to have subaged. A $\mu>1$ is called superaging and $\mu=1$ corresponds to full aging. For TRM experiments a $\mu$ of approximately 0.9 is found for different types of spin glasses 4,5$]$, over a wide range of reduced temperatures indicating subaging. At very low temperatures and temperatures approaching the transition temperature, $\mu$ is observed to decrease from the usual 0.9 value. Superaging has been observed in Monte Carlo simulations of spin glasses [6]. This has led to confusion as to the exact nature of scaling.
Zotev et al. 7] have suggested that the departures from full $\frac{t}{t_{w}}$ scaling, observed in aging experiments, are mainly due to cooling effects. In a real experimental environment, the situation is complicated by the time it takes for the sample to cool to its measuring temperature. An effect due to the cooling rate at which the sample temperature approaches the measuring temperature has been known [8, 9]. This effect is not trivial, it does not contribute a constant time to $t_{w}$.

Another possible explanation for the deviation from full aging comes from the widely held belief that the magnetization decay is an additive combination of a stationary term $\left(M_{\text {Stat }}=A\left(\tau_{0} / t\right)^{\alpha}\right)$ and an aging term $\left(M=f\left(\frac{t}{t_{w}}\right)\right)[10,11,12]$. Subtraction of a stationary term, where $\tau_{0}$ is a microscopic spin flipping time, A is a dimensionless constant and $\alpha$ is a parameter determined from $\chi^{\prime \prime}$ measurements, was shown to increase $\mu$ from 0.9 to 0.9712 .

In this letter we analyze effects of the cooling time through a series of different cooling protocols and we present the first clear and unambiguous experimental evidence that the TRM decays scale as $\frac{t}{t_{w}}$ (i.e. full aging).

Three different methods have been regularly employed to understand the scaling of the TRM decays. The first and simplest is to scale the time axis of the magnetization decay with the time the sample has spent in a magnetic field (i.e. $\frac{t}{t_{w}}$ ) [3] . If the decays scale as a function of waiting time it would be expected that the decay curves would overlap. This has not yet been observed.

A second more sophisticated method was initially developed by Struik 13] for scaling the dynamic mechanical response in glassy polymers and first applied to spin glasses by Ocio et al. [5]. This method plots the log of the 

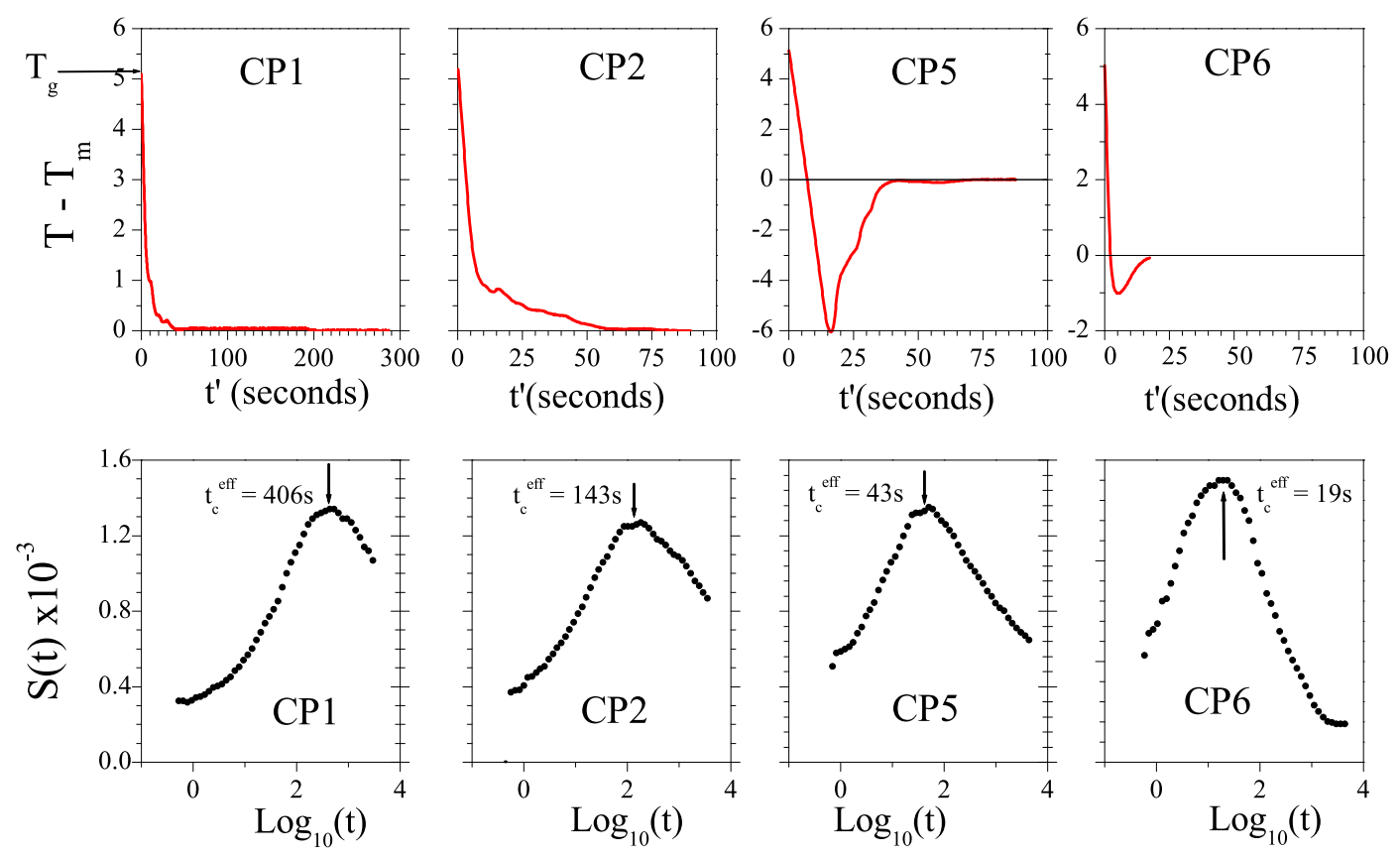

FIG. 1: The cooling time(t') start when $T_{g}$ is crossed. For CP1-2 the temperature is never dropped below $T_{m}$. On the contrary for CP5-6 the temperature is not allowed to go above $T_{m}$. The effective cooling time, $t_{c}^{e f f}$ is associated with a peak in the ZTRM S(t). The $\mathrm{S}(\mathrm{t})$ for each each cooling protocol are plotted with the the same y-scale.

reduced magnetization $M / M_{f c}\left(M_{f c}\right.$ is the field cooled magnetization), against an effective waiting time $\xi=\frac{\lambda}{t_{w}^{\mu}}$ where

$$
\lambda=\frac{t_{w}}{1-\mu}\left[\left(1+\frac{t}{t_{w}}\right)^{1-\mu}-1\right] ; \quad \mu<1
$$

or

$$
\lambda=t_{w} \log \left[1+\frac{t}{t_{w}}\right] ; \quad \quad \mu=1
$$

A value of $\mu=1$ would correspond to perfect $t / t_{w}$ scaling. Previous values of $\mu$ obtained on the decays using this method have varied from .7 to .94[4] for a temperature range $.2<T_{r}<.95$. A value of $\mu<1$ is called subaging.

Finally a peak in the function $\mathrm{S}(\mathrm{t})$

$$
S(t)=-\frac{1}{H} \frac{d M(t)}{d\left[\log _{10}(t)\right]}
$$

as a function of time has been shown to be an approximately linear function of the waiting time 2]. This peak occurs at a time slightly larger then the waiting time, again suggesting possible subaging.

In this study we use all three of the above scaling procedures to analyze the data we have produced with different cooling protocols. All measurements in this letter were performed on our homebuilt DC SQUID magnetometer with a $C u_{.94} M n_{.06}$ sample. The sample is well documented 14] and has been used in many other studies. The measurements described in this letter were performed in the following manner: The sample was cooled, in a magnetic field of $20 \mathrm{G}$, from $35 \mathrm{~K}$ through its transition temperature of $31.5 \mathrm{~K}$ to a measuring temperature of $26 \mathrm{~K}$. This corresponds to reduced temperature of .83 $T_{g}$. The sample was held at this temperature for a waiting time $t_{w}$, after which time the magnetic field was rapidly decreased to $0 \mathrm{G}$. The resulting magnetization decay is measured 1s after field cutoff to a time greater than or equal to $5 t_{w}$. The only parameters we have varied in this study are $t_{w}$ and the rate and profile at which we cool the sample through the transition temperature to the measuring temperature. The sample is located on the end of the sapphire rod and sits in the upper coil of a second order gradiometer configuration. The temperature measuring thermometer is located $12.5 \mathrm{~cm}$ above the sample. Heat is applied to the sample through a heater coil located on the same sapphire rod $17 \mathrm{~cm}$ above the sample. Sample cooling occurs by heat transfer with the He bath via a constant amount of He exchange gas, which was previously introduced into each chamber of the double vacuum jacket. We have measured the decay time of our field coil and find that we can quench the field in less then $0.1 \mathrm{~ms}$. We have also determined that without a sample, our system has a small reproducible exponential decay, that decays to a constant value less than the system noise within 400 seconds. In order to accurately describe our data we subtract this system decay from all of the data.

In this paper we present TRM data for eight waiting 




FIG. 2: In the first column the TRM decays curves for $t_{w}=$ $50 \mathrm{~s}, 100 \mathrm{~s}, 300 \mathrm{~s}, 1000 \mathrm{~s}, 3600 \mathrm{~s}, 6310 \mathrm{~s}, 10000 \mathrm{~s}$ are plotted for the different cooling protocols. In the middle column the decays are scaled with the waiting. The y-axis for columns one and two are the same. The decays collapse onto each other as $t_{c}^{e f f}$ decreases. In the third column the decays are scaled with $\mu$. Where $\lambda$ from eq 1 one is used.

times $\left(t_{w}=50 \mathrm{~s}, 100 \mathrm{~s}, 300 \mathrm{~s}, 630 \mathrm{~s}, 1000 \mathrm{~s}, 3600 \mathrm{~s}, 6310 \mathrm{~s}\right.$, and 10000s). The same TRM experiments were performed for six different cooling protocols. In this paper we use four of the cooling protocols. Figure 1 (top row) is a plot of temperature vs. time for four of the cooling protocols. These different cooling protocols, were achieved, by varying applications of heat and by varying the amount of exchange gas in the vacuum jackets. A more detailed description of the cooling protocols will be given in a followup publication. In Figure 1 (bottom row) we plot $\mathrm{S}(\mathrm{t})(\mathrm{Eq}$ 3) of the ZTRM protocol (i.e. zero waiting time TRM) in order to characterize a time associated with the cooling protocol, $t_{c}^{e f f}$. As observed in Figure 1 we have achieved effective cooling times ranging from 406s down to 19 seconds. These times can be compared with commercial magnetometers which have cooling times in the range of 100-400s.

In Figure 2, we plot the data for the TRM decays (first column) with the four cooling protocols. It should be noted that the magnetization (y-axis) is scaled by the field cooled magnetization.

The second column is the same data, as column one, with the time axis(x-axis) normalized by $t_{w}$. It can be observed for $\frac{t}{t_{w}}$ scaling (column 2) that as the effective cooling time decreases the spread in the decays decreases giving almost perfect $\frac{t}{t_{w}}$ scaling for the 19 second cooling protocol.

The last column in Figure 2, is the data scaled,using $\mu$ scaling which has previously been described. It has long been known that the rate of cooling affected $\mu$ scaling and that $\mu$ scaling is only valid in the limit $t_{w}>>t_{c}^{e f f}$. We find this to be true and that the limit is much more rigorous than previously believed. To determine $\mu$ scaling, we focused on applying this scaling to the longest waiting time data (i.e., $t_{w}=3600 \mathrm{~s}, 6310 \mathrm{~s}$ and $10,000 \mathrm{~s}$ ). For the largest effective cooling time data, $t_{c}^{e f f}=406 s$, we find that we can fit the longest waiting time data with a $\mu$ value of .88 . This is consistent with previously reported values of $\mu[4]$. We do find, however, that TRM data with waiting times less then 3600 s do not fit on the scaling curve. We find that scaling of the three longest waiting time decays produces $\mu$ values which increase as $t_{c}^{e f f}$ decreases. We also find that as $t_{c}^{e f f}$ decreases, the data with shorter $t_{w}$ begins to fit to the scaling better. It can be observed that at $t_{c}^{e f f}=19 \mathrm{~s}$ we obtain almost perfect scaling for all of the data with a value of $\mu=.999$. However we find we can reasonably fit the data to a range of $\mu$ between .989-1.001. The fitting for the large $t_{w}$ decay curves, $t_{w}=3600 \mathrm{~s}, 6310 \mathrm{~s}$ and $10,000 \mathrm{~s}$, is very very good. Small systematic deviations, as a function of $t_{w}$, occur for $t_{w}<3600 \mathrm{~s}$ with the largest deviations for $t_{w}=50 \mathrm{~s}$. Even with an effective cooling time two orders of magnitude less then the waiting time, one sees deviations from perfect scaling. We have also scaled the data using Eq. 2. We find no noticeable difference between the quality of this fit and the quality of the $\mu=.999$, for $t_{c}^{e f f}=19 \mathrm{~s}$, shown in Figure 2. Data with longer cooling times cannot be fit with Eq. 2. We therefore conclude that full aging is observed for the long $t_{w}$ data using the $t_{c}^{e f f}=19 \mathrm{~s}$ protocol.

It is clear from Figure 1 (bottom row), that the effect of the cooling time has implications for the decay all the way up to the longest time measured, 10,000 seconds. The form of the $\mathrm{S}(\mathrm{t})$ of the ZTRM is very broad. The $\mathrm{S}(\mathrm{t})$ function is often thought of as corresponding to a distribution of time scales (or barrier heights) within a system which has infinite equilibration times (barriers). The peak in $\mathrm{S}(\mathrm{t})$ is generally associated with the time scale (barrier height) probed in time $t_{w}$. In Figure 1, we observe that for the larger effective cooling times, the waiting times correspond to points on or near the peak of $\mathrm{S}(\mathrm{t})$ for the ZTRM. We therefore believe that for the larger effective cooling times there is significant contamination from the cooling protocol over the entire region of $t_{w} \mathrm{~s}$ used in this paper. Only for $t_{c}^{e f f}=19 \mathrm{~s}$ cooling protocol do we find that the majority of $t_{w} s$ occur far away from the peak in the $\mathrm{S}(\mathrm{t})$.

All the data in figure 3 used the cooling protocol with $t_{c}^{e f f}=19 s$. In Figure 3a we plot the $\mathrm{S}(\mathrm{t})(\mathrm{ZTRM})$ for $t_{c}^{e f f}=19 s$ with arrows to indicate the waiting times for the TRM measurements. It can be observed that after approximately 1000 seconds the slope of the $\mathrm{S}(\mathrm{t})$ function decreases, possibly approaching a horizontal curve, which would correspond to a pure logarithmic decay in $\mathrm{M}(\mathrm{t})$. If, 

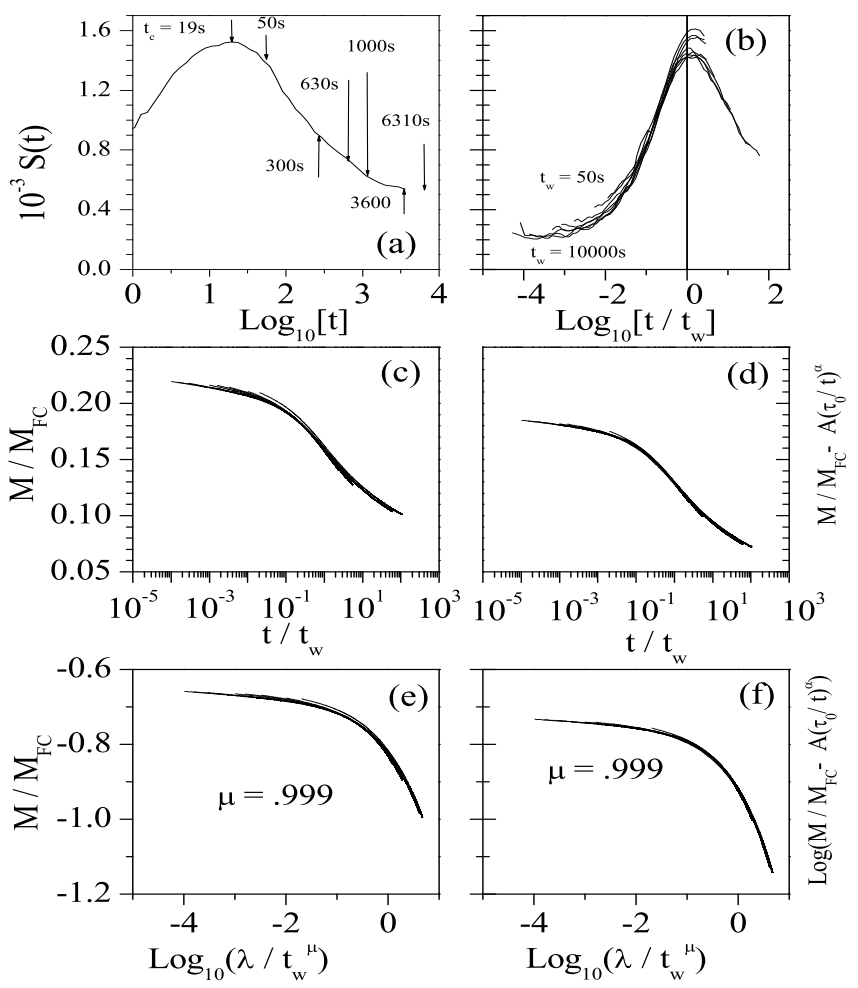

FIG. 3: The $\mathrm{S}(\mathrm{t})(\mathrm{ZTRM})$ for $t_{c}=19 \mathrm{~s}$ is shown with the different $t_{w} \mathrm{~s}$ marked(a). In figure b. the $\mathrm{S}(\mathrm{t})$ for all $\operatorname{decays}\left(t_{c}^{e f f}=19 s\right)$ are scaled with $t_{w}$. The decay curves are scaled with $t_{w}(\mathrm{c})$ and $\mu$-Scaled(e), compared to the same scaling for decays with a stationary part subtracted(d and $\mathrm{f})$. For stationary part we use $\mathrm{A}=0.06, \alpha=0.02$ and $\tau_{0}=10^{-12}$

on the other hand, the slope is continuously changing this part of the decay may be described by a weak power law. Either way, this region would correspond to aging within a pure non-equilibrated state. We believe that the long waiting time data occurs outside the time regime that has been corrupted by the cooling time and that this is the reason that we have, for the first time, observed full aging.

It has been suggested that subtraction of a stationary component of the magnetization decay will improve scaling [12. The very long time magnetization decay is believed to consist of a stationary term that is thought to decay as a power law. We fit a power law, $M(t)=$ $A\left(\tau_{o} / t\right)^{\alpha}$ to the long time decay $(1000 \mathrm{~s}-5000 \mathrm{~s})$, of the ZTRM for $t_{w}=19 \mathrm{~s}$. Using $\tau_{o}=10^{-12} s$, we find $\alpha=.07$ and $A=.27$. Subtracting this power law form from the magnetization decay destroys scaling. We find that the subtraction of a much smaller power law term with $\mathrm{A}=.06$ and $\alpha=.02$ slightly improves scaling at both short and long times. While the $\alpha$ values for the two different power law terms we have fit to are quite different, both values fall within the range determined from the decay of $\chi$ "12]. In Figure 3(c-d) and 3(e-f), we plot the two different types of scaling we have performed, with and without the subtraction of the weaker power law term.

We find that even for $t_{c}^{e f f}=19 s$ the peak in $\mathrm{S}(\mathrm{t})$ for $t_{w}>1000 s$ occur at a time larger then $t_{w}($ fig $3 \mathrm{~b})$. We find that we can fit the effective time associated with the peak in $\mathrm{S}(\mathrm{t})$ to $t_{w}^{e f f}=t_{w}^{1.1}$.

In summary, we have performed TRM decays over a wide range of waiting times (50s - 10,000s) for six different cooling protocols. We find that as the time associated with the cooling time decreases, scaling of the TRM curves improves in the $\frac{t}{t_{w}}$ scaling regime and in the $\mu$ scaling regime. In $\mu$ scaling we find that as the effective cooling time decreases $\mu$ increases approaching a value of .999 for $t_{c}^{e f f}=19 s$. For the $t_{c}^{e f f}=19 s$ TRM decays, we find that subtraction of a small power law term $(\mathrm{A}=.06$, $\alpha=.02$ ) slightly improves the scaling. It is however likely that the small systematic deviations of the $t_{c}^{\text {eff }}=19 \mathrm{~s}$ data as a function of $t_{w}$ are associated with the small but finite cooling rate.

The authors would like to thank V. S. Zotev, E. Vincent and J. M. Hammann for very helpful discussions.

[1] R. V. Chamberlin, M. Hardiman and R.Orbach, J. Appl. Phys 52, 1771 (1983).

[2] L.Lundgren, P.Svedlindh, P.Nordblad and O.Beckman, Phys. Rev. Lett. 51, 911(1983); L.Lundgren, P.Svedlindh, P.Nordblad and O.Beckman, J. Appl. Phys. 57, 3371 (1985).

[3] J.P. Bouchaud, J. Phys. I (Paris) 2,1705 (1992).

[4] M. Alba, M. Ocio and J. Hammann, Euro Phys Lett, 2, 45 (1986); M. Alba, J. Hammann, M. Ocio, Ph. Refregier and H.Bouchiat, J. Appl. Phys 61, 3683 (1987).

[5] M. Ocio, M. Alba,J. Hammann, J. Phys. (PARIS) Lett. 46, 1101 (1985)

[6] Private communication Pablo Sibani

[7] V. S. Zotev, G. F. Rodriguez, G. G. Kenning, R. Orbach, E. Vincent and J. Hammann, cond-matt/0202269 To be published in Phys Rev B.

[8] P. Nordblad, P. Svedlindh, L. Sandlund and L.Lundgren,Phys Lett A 120, 475 (1987).

[9] K. Jonason, P. Nordblad, Physica B 279, 334 (2000).

[10] L. F. Cugliandolo and J. Kurchan, J. Phys A27, 5749 (1994).

[11] J. P. Bouchaud and D. S. Dean, J.Phys. I (Paris) 5, 265 (1995).

[12] E.Vincent, J.Hammann, M.Ocio, J.P.Bouchaud and L.F. Cugliandolo, Slow dynamics and aging in spin glasses. Complex behaviour of glassy systems, ed. M. Rubi, Sitges conference, can be retrieved as cond-matt/9607224

[13] L.C.E Struik, Physical Ageing in Amorphous polymers and other materials Lesevier Sci Pub Co. Amesterdam 1978

[14] G. G. Kenning, D. Chu, R. Orbach, Phys. Rev. Lett 66, 2933 (1991) 